\title{
tirants d'ancrage permanents forés injectés dans le sol du type INTRAPAC utilisant le procédé des barres précomprimées
}

\author{
L. MASTIKIAN \\ Directeur des études de la Société INTRAFOR COFOR* \\ A. FUENTES \\ Maître de conférences à l'Ecole Nationale des Ponts et Chaussées**
}

\section{DESCRIPTION DU PROCÉDÉ INTRA- PAC DE TIRANT DÉFINITIF FORÉ ET INJECTÉ DANS LE SOL}

L'utilisation du procédé «barres précomprimées dans le cadre du béton précontraint est déjà connue et répandue. Une barre précomprimée se présente sous forme d'une gaine métallique, d'un diamètre variable de 40 à $100 \mathrm{~mm}$ constituée d'un feuillard de $2 / 10$ à $6 / 10 \mathrm{~mm}$ d'épaisseur. A l'intérieur de cette gaine, on injecte un coulis après y avoir tendu des fils, tels que ceux utilisés pour la précontrainte à fils adhérents, jusqu'à une contrainte proche de la limite élastique.

Lorsque, après durcissement du coulis, les fils de précontrainte sont détendus, la contrainte de compression dans le coulis durci se situe alors entre 25 et $30 \mathrm{MPa}$, ce qui représente sensiblement la moitié de la contrainte de rupture du coulis durci et fretté. Sous une telle contrainte, la barre précomprimée subit un raccourcissement instantané, puis un raccourcissement de fluage pendant plusieurs mois. Si dans un délai assez court, après fabrication ( 1 mois par exemple),

$\left.{ }^{*}\right)$ 15, rue des Sablons - 75016 Paris.

(**) 28, rue des Saints-Pères - 75007 Paris. cette barre est logée dans la cage d'armatures d'un élément bétonné « in situ * ou préfabriqué, le fluage de la barre contribue à comprimer le béton de complément qui entoure cette barre: ce béton de complément se trouve ainsi partiellement précontraint.

Ainsi, l'utilisation de barres précomprimées dans une pièce en béton, soumise à une sollicitation de flexion ou de traction, permet de retarder la fissuration et de diminuer la déformation tout en assurant une excellente tenue au feu et une excellente protection contre les agents agressifs, du fait de l'enrobage des aciers de précontrainte, et puisque la phase de décompression du coulis entourant les câbles n'est jamais atteinte.

Dès lors, il devenait extrêmement tentant de remplacer dans un tirant foré injecté le faisceau de câbles de précontrainte par une barre unique selon le procédé " précomprimé». C'est le principe du tirant définitif du type INTRAPAC.

\section{Protection des fils de précontrainte}

Ces fils, incorporés dans la barre, seront en permanence entourés par une gaine de coulis en état de forte compression donc non fissurée, empêchant tout contact du milieu environnant, et surtout de l'eau avec 
l'acier. Même si le tube acier entourant le coulis et les câbles, bien que protégé par un produit du type goudron ou brai-epoxy, vient à se corroder â terme, les produits de décomposition, oxyde de fer, sont encore capables de transmettre les efforts de cisaillement du terrain au cylindre de coulis qui, lui, reste toujours en état de forte compression.

\section{Suppression éventuelle de la mise en tension préalable des tirants d'ancrage}

Elle est réalisée au moyen d'un vérin hydraulique. En effet, la charge utile de la barre étant reprise par un allongement unitaire inférieur à $1 \%$, l'allongement de la partie libre du scellement, long de 10 à $20 \mathrm{~m}$ pour les ouvrages courants, serait dans la fourchette de 1 à $2 \mathrm{~cm}$ donc tout à fait comparable au déplacement des têtes d'ancrage de tirants précontraints, consécutifs à leur surtension quand la structure est mise en charge.

Il s'agit d'un aspect qui est propre aux tirants du type "passif».

Cela évite la phase dite de "mise en tension" qui nécessite dans certains cas un surdimensionnement de l'ouvrage.

On laisse ainsi l'ouvrage libre de développer la réaction nécessaire pour sa stabilité, tout en restant dans les limites d'un déplacement acceptable. On évite de solliciter inutilement louvrage de soutènement, par exemple, au moyen de forces qui résultent de calculs théoriques le plus souvent très sécurisants, et qui n'existent pas toujours dans la réalité à moins qu'on ne les provoque par des mises en tension intempestives des tirants.

Néanmoins, il est toujours possible de mettre en tension et de relâcher partiellement un certain échantillonnage de tirants afin de s'assurer que les scellements tiennent.

Simplification extrême du dispositif de protection des câbles

Le tirant a le même profil suivant toute sa longueur. On est loin des tirants traditionnels comportant successivement: câbles à nu suivant la longueur du scellement, câbles à protection souple dans la partie libre, à nouveau câbles à nu pour accrochage sur les clavettes et la plaque.

Les raccordements entre ces divers tronçons des tirants traditionnels constituent autant de points faibles en matière de corrosion, sans compter leur coût et les soins nécessaires pour leur réalisation et leur mise en œuvre.

En ce qui concerne les tirants INTRAPAC, et cela a été mis au point jusqu'à des capacités utiles de $550 \mathrm{kN}$ (soit $4,15 \mathrm{t}$ ), l'accrochage de tête du tirant se fait au moyen d'une clavette de grand diamètre s'agrippant sur le tube extérieur sans aucune nécessité de mettre les câbles à nu.
Ce dispositif de tête permet par ailleurs la suppression des chaises d'appui classiques, du fait de l'inclusion de la pièce femelle de la clavette dans l'épaisseur de louvrage (figs. 1,2 et 3 ).

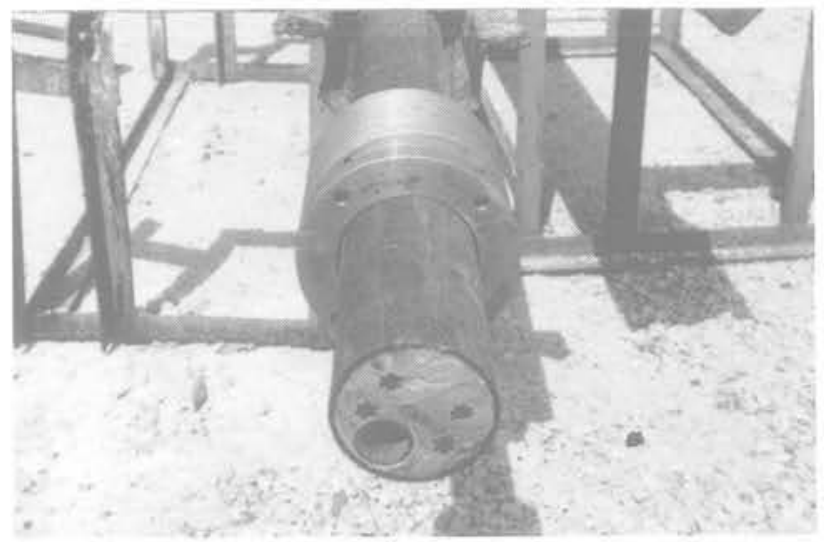

Fig. 1. - Coupe d'une barre et dispositif d'accrochage.

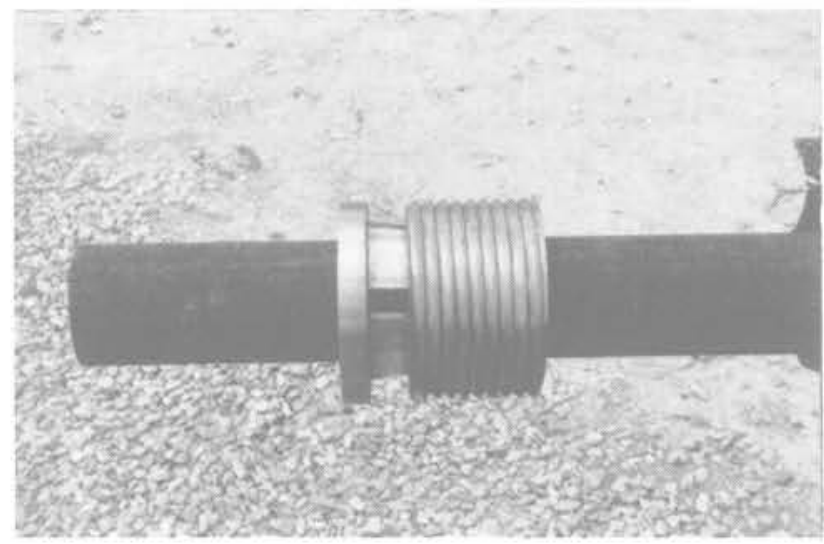

Fig. 2. - Dispositif d'accrochage monté sur la barre.

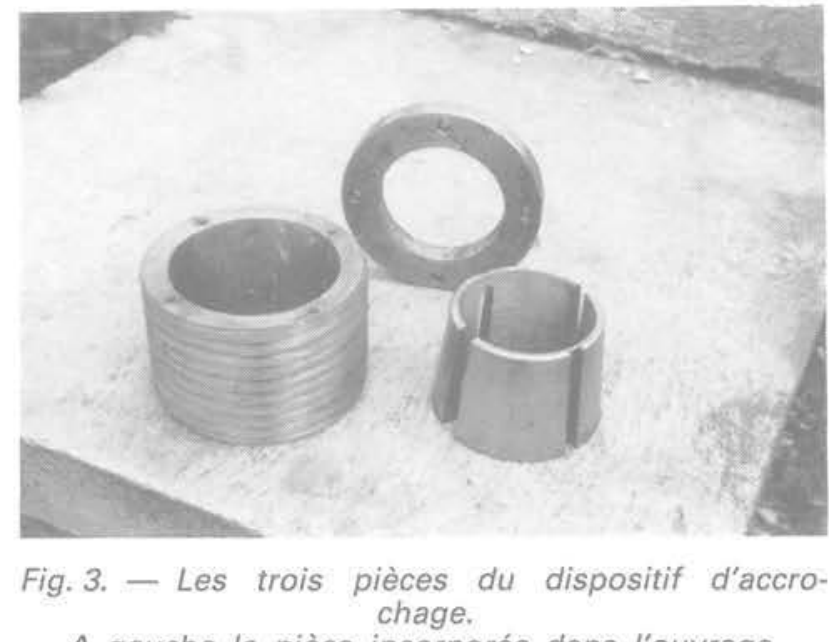

A gauche la pièce incorporée dans l'ouvrage. 


\section{Préfabrication des barres en atelier}

Elle facilite le contrôle permanent et rigoureux garantissant la qualité des barres.

L'utilisation du procédé "barres précomprimées" pour la mise au point des tirants du type INTRAPAC, a néanmoins posé un certain nombre de problèmes dont nous allons énumérer les principaux.

A l'heure actuelle, des tirants d'une capacité utile de $550 \mathrm{kN}(4,15 \mathrm{t})$ ont été réalisés et mis en cuvre sur le site. La mise au point de tirants d'une capacité utile proche de $100 \mathrm{t}$, ne devrait constituer qu'un problème d'extrapolation.

\section{SCELLEMENT DE LA BARRE AU TERRAIN}

Pour les terrains de forte raideur (roche, roche tendre, argiles très compactes) qui ne nécessitent pas un scellement injecté, la barre est utilisée dans les mêmes conditions que tout autre type de tirant. Le forage est rempli au moyen d'un coulis épais de ciment à forte résistance et la barre est ensuite introduite dans le forage. Selon le comportement de l'ouvrage et du massif de terrain environnant, la longueur libre de la barre se crée par fissuration du coulis dans les zones proches de la structure, c'est-à-dire suivant le tronçon du tirant situé dans la largeur du cône d'éboulement.

Dans ce genre d'applications, (cloutage de talus, de radiers soumis à des sous-pressions, micro pieux sollicités alternativement en compression et traction), la barre se satisfait de forages d'un diamètre comparable à celui de tout autre type de tirant. Elle garde néanmoins les avantages que nous venons d'énumérer.

Dans l'éventualité de terrains meubles (sables compacts ou lâches, argiles moyennement compactes et compressibles) qui nécessitent une injection préalable à forte pression afin d'assurer le scellement du tirant, la solution suivante a été mise au point.

Un tube d'injection de 2,5 ou $3,75 \mathrm{~cm}$ de diamètre, en matière plastique, est placé à l'intérieur de la barre au moment de la fabrication comme indiqué sur le schéma et photos ci-joints (figs. 1, 4 et 5).

Des ouvertures sont percées à travers les épaisseurs du tube extérieur et du tube d'injection et protégées par des manchettes caoutchouc placées autour de la barre. Ce tube extérieur peut être embouti ou équipé par des spirales d'acier soudées afin d'améliorer son adhérence au coulis.

Le scellement de la barre au terrain se fait alors par injection à travers le tube ainsi placé, en utilisant un double obturateur.

Ce dispositif, essayé sur le chantier, a donné entière satisfaction.

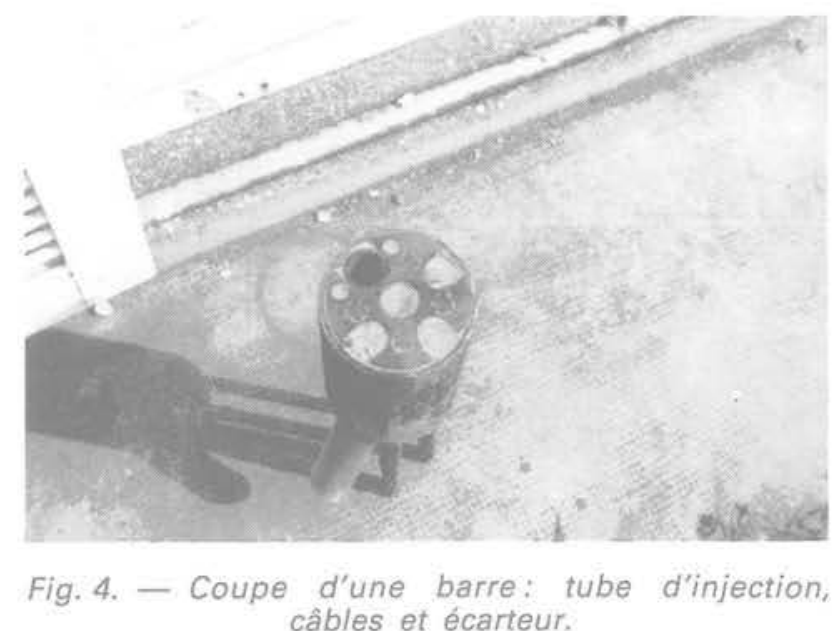

câbles et écarteur.

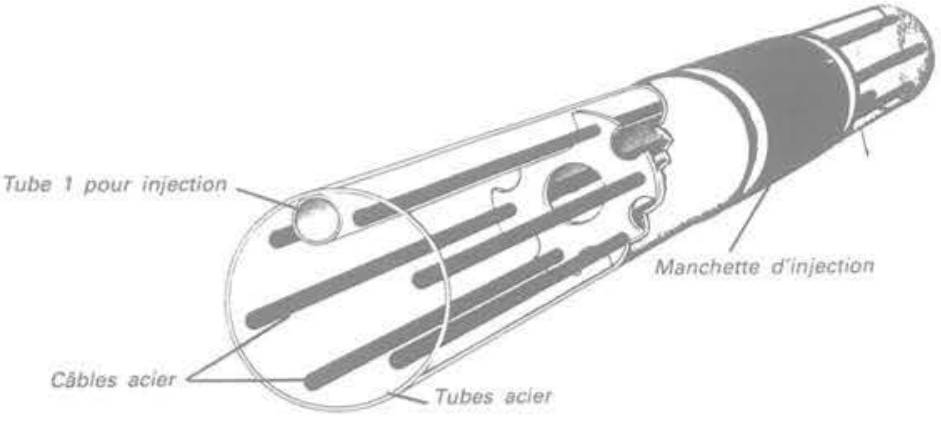

Fig. 5. - Vue écorchée d'une barre INTRAPAC.

\section{CHOIX DU DIAMẼTRE \\ DU TUBE EXTÉRIEUR \\ EN FONCTION DE LA CHARGE UTILE DU TIRANT}

Ce choix doit satisfaire aux deux exigences suivantes:

- Bonne tenue de la barre INTRAPAC lors du relâchement des câbles. Bien qu'en théorie, une pièce précomprimée par fils adhérents ne craigne pas une rupture par flambement, le procédé a des limites que nous avons observées par des essais successifs en utilisant pour une force donnée de précontrainte des tubes de diamètres décroissants. Ainsi, pour $6,15 \mathrm{t}$ tendus à la limite élastique, soit plus de $1300 \mathrm{kN}$ dans un tube $114-107 \mathrm{~mm}$, le relâchement s'est traduit par une courbure excessive du tube qui l'a rendu inutilisable dans un forage. Les irrégularités de positionnement des câbles par rapport à la section comprimée créent une densité de flexion le long de la barre dont la section et l'inertie insuffisantes se traduisent par des courbures excessives. 
Suite à une série d'essais, les diamètres suivants se sont avérés satisfaisants (fig, 6):

$\varnothing 114-107 \mathrm{~mm}$ pour $4,15 \mathrm{t}$ soit une charge utile de $550 \mathrm{kN}$

$\varnothing 76-70 \mathrm{~mm} 6$ fils $7 \mathrm{~mm}$ soit une charge utile de $250 \mathrm{kN}$

$\varnothing 102-94 \mathrm{~mm} 4,13 \mathrm{t}$ soit une charge utile de $400 \mathrm{kN}$.



Fig. 6. - Essai de traction simple. Courbe effort déformation d'une barre.

barres: tube extérieur $\varnothing 76,70 \mathrm{~mm}$

câbles: $6 \varnothing 7 \mathrm{~mm}$

Pour $6.15 \mathrm{t}$, charge utile de $850 \mathrm{kN}$, un $\varnothing 140-132$ devrait contenir bien que l'essai n'ait pas encore été réalisé.

- La deuxième exigence est un forage du plus faible diamètre possible pour des raisons évidentes d'économie, de rapidité et de commodité. Par ailleurs, compte tenu de la rigidité de la barre, ce forage doit accuser le moins possible de courbure afin de faciliter l'introduction de la barre. La solution la mieux adaptée est le forage à la boue et au tricône utilisant un train de tiges de forte inertie d'un diamètre très proche de celui du forage. Compte tenu du $\varnothing$ de la barre, majoré des excroissances des manchettes, des diamètres de forage de près de $200 \mathrm{~mm}$ sont d'ores et déjà à envisager pour des capacités utiles de $1000 \mathrm{kN}$.

\section{MANUTENTION DE LA BARRE INTRAPAC}

La forte raideur de la barre nécessite l'utilisation d'un palonnier en profilé métallique, avec des attaches sur la barre espacées d'une dizaine de mètres. Le chantier est équipé d'une grue légère sur pneus, le poids d'une barre de $30 \mathrm{~m}$ d'une capacité de $1000 \mathrm{kN}$ utile ne dépassant pas la tonne. Ce qui est perdu en amortissement de matériel se retrouve largement en rapidité et qualité d'exécution (figs. 7 et 8 ).

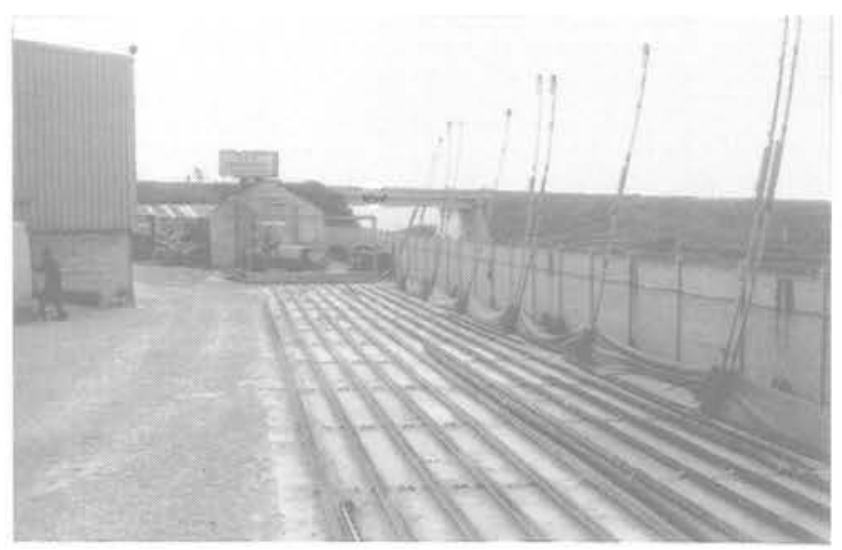

Fig. 7. - Aire de préfabrication des barres INTRAPAC.

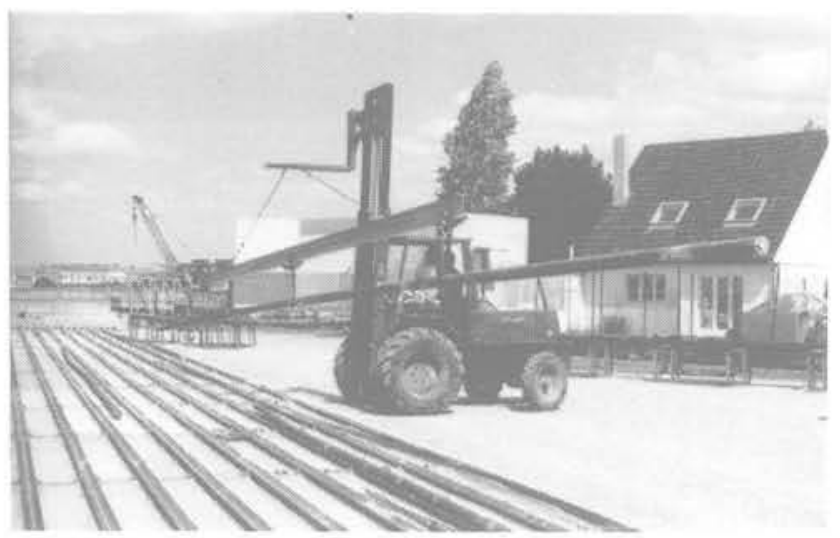

Fig. 8. - Manutention d'une barre INTRAPAC.

La forte raideur de la barre, quoique gênante pour les manutentions d'approche, facilite énormément les opérations d'introduction dans le forage.

Le forage et l'équipement d'un tirant de $18 \mathrm{~m}$ de long ont nécessité une durée de 1 h 30 . Le foreur et deux aides, en plus du grutier, ont suffi pour mettre la barre en place.

\section{CHOIX DU COULIS DE REMPLISSAGE DE LA BARRE INTRAPAC}

Les recherches se sont orientées de manière à obtenir les coulis les plus résistants dans le laps de temps le plus court, de manière à libérer au plus tôt l'aire de préfabrication. Par ailleurs, les modules élastiques de 
ces coulis doivent être les plus élevés possible afin d'éviter un relâchement excessif de la tension des câbles lors de leur détente (fig. 9).

Les coulis utilisant essentiellement du sable SIFRACO, du ciment et divers types d'adjuvants répondent largement aux exigences du procédé. Ainsi, on obtient à trois jours des résistances de plus de $60 \mathrm{Mpa}$ et $\mathrm{E}=$ $35000 \mathrm{Mpa}$ à court terme, et à $10 \mathrm{j}$, les résistances dépassent facilement les $100 \mathrm{Mpa}$ avec $\mathrm{E}=$ $23000 \mathrm{Mpa}$ à long terme.

Par ailleurs, ces coulis accusent une rhéologie suffisante pour permettre le remplissage des tubes de grandes longueurs, 20 à $50 \mathrm{~m}$ dont la section est encombrée par les cấbles, le tube d'injection et les nombreux écarteurs.

Le ressuage des coulis, c'est-à-dire la décantation avec apparition d'eau dans la partie haute de l'échantillon, a été vérifiée tout particulièrement à l'intérieur du tube de la barre par des contrôles aux rayons X.

\section{ESSAIS RÉALISÉS AVEC DES BARRES INTRAPAC}

Des essais ont été réalisés au quai Bougainville du Port Autonome du Havre en septembre 1982.

Deux barres INTRAPAC de 4,13t, tubes extérieurs en acier doux, $\varnothing 101,6 \mathrm{~mm}-94,4 \mathrm{~mm}$ et d'une longueur de $16,5 \mathrm{~m}$ ont été mises en œuvre.
Ces essais ont comporté toutes les phases courantes de la réalisation des tirants d'ancrage:

- forage et remplissage au moyen d'un coulis riche en ciment:

- mise en place des barres équipées de tubes à manchettes;

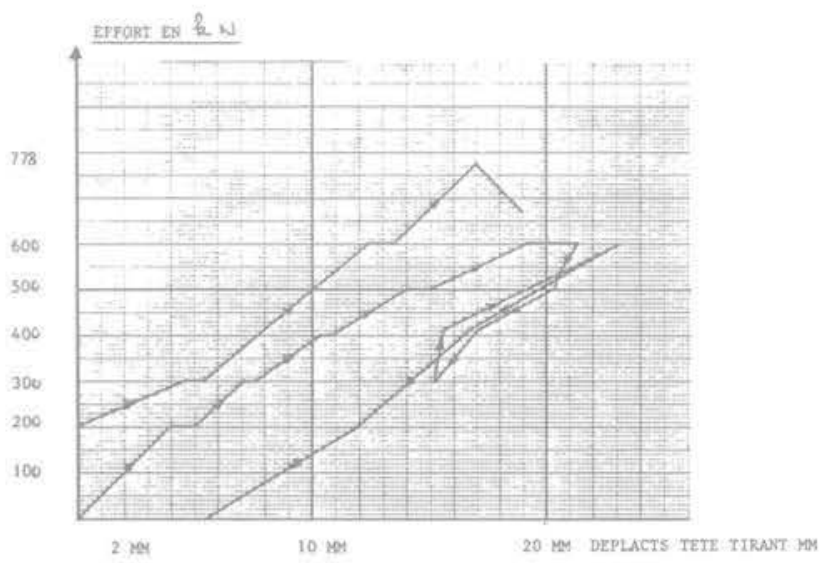

Fig. 10, - Courbe du tirant d'essai en barre INTRAPAC.

Port Autonome du Havre, septembre 1982.

- tube extérieur $\varnothing 102-94 \mathrm{~mm}$

- câbles 4,13t

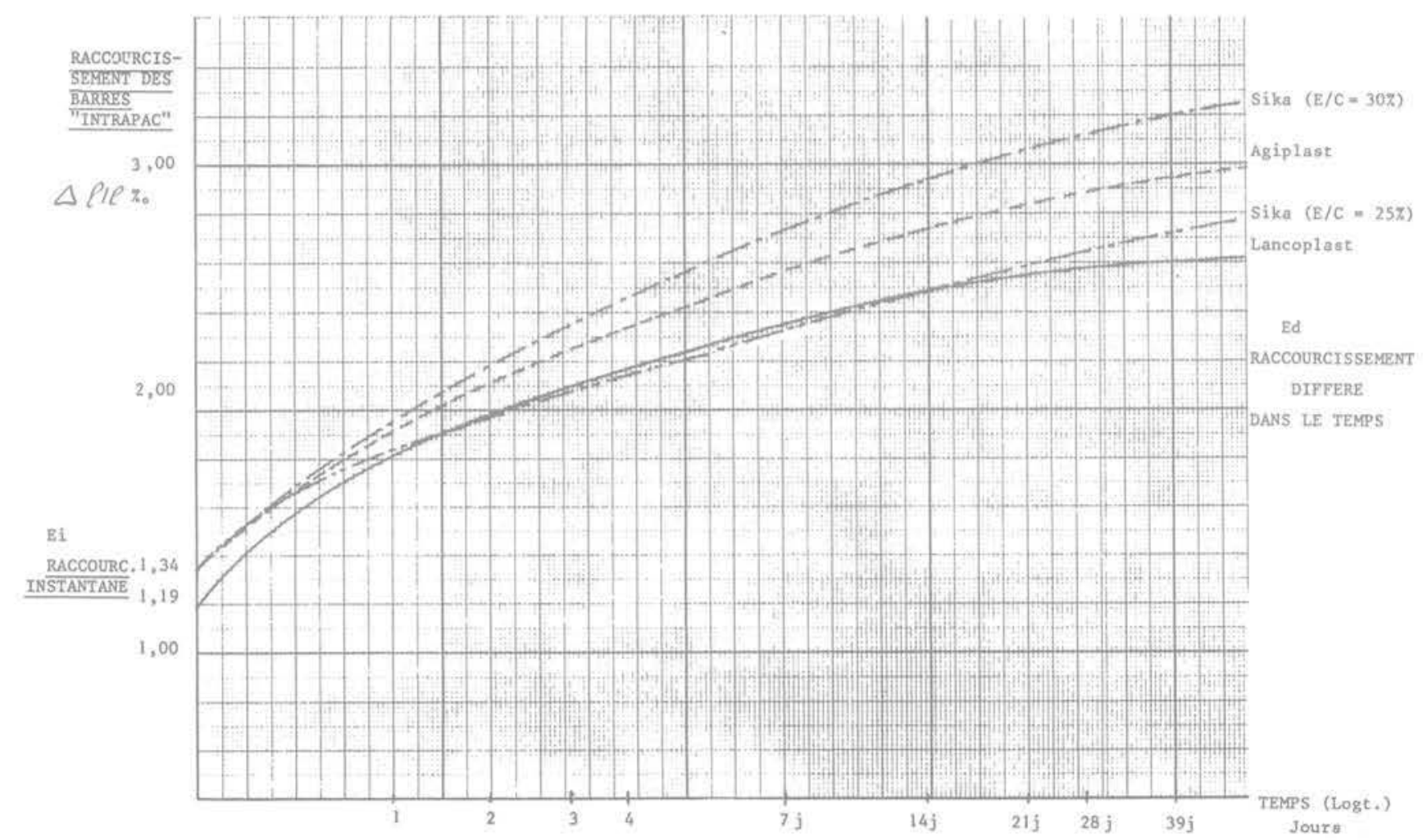

Fig. 9. - Raccourcissement instantané et différé des barres "INTRAPAC" suivant composition des mortiers de remplissage. 
- injection sous pression dans la zone de scellement à travers les tubes plastiques prévus à l'intérieur des barres;

- mise en tension des barres jusqu'à la rupture après durcissement des scellements.

Les courbes déplacement tête du tirant en fonction de l'effort dans le tirant sont données en $\mathrm{kN}$ dans le graphique de la figure 10 .

Le premier de ces tirants a cédé à $780 \mathrm{kN}$, et le deuxième à $690 \mathrm{kN}$

On peut constater, compte tenu de l'allure de ces courbes, que la rupture s'est produite par insuffisance du scellement: glissement de la barre. En effet, du fait de leur faible longueur, ces barres étaient scellées dans des formations d'une compacité insuffisante: remblais hydrauliques inconsistants et argiles silteuses surmontant le toit des sables verts compacts que l'on trouve couramment dans cette zone portuaire.

La capacité utile calculée pour ces tirants était de $400 \mathrm{kN}$

Les barres INTRAPAC se sont comportées de manière satisfaisante par elles-mêmes. Une légère concavité des courbes effort $\mathrm{x}$ allongement dénote, pour les charges élevées, une diminution de la section de la barre. Cela est dâ à la limite élastique $(215 \mathrm{Mpa})$ atteinte dans le tube extérieur, et peut-être à la mise en état de traction du coulis entourant les câbles.

\section{CONCLUSION}

Les principaux avantanges du procédé sont les suivants:

- une protection totale des fils de précontrainte incorporés dans la barre. En effet, ces fils seront en permanence entourés par une gaine de mortier en état de compression, donc non fissurée, empêchant tout contact du milieu environnant avec l'acier;

- la suppression éventuelle de mise en tension des tirants au moyen d'un vérin hydraulique;

- une simplification extrême du dispositif de protection des câbles, le tirant ayant le même profil sur toute sa longueur.

L'accrochage de tête se fait au moyen d'une clavette de grand diamètre s'agrippant sur le tube extérieur sans nécessité aucune de mettre les cábles à nu. 